\title{
Tricuspid Valve Disorder
}

National Cancer Institute

\section{Source}

National Cancer Institute. Tricuspid Valve Disorder. NCI Thesaurus. Code C78649.

A heart disorder characterized by a defect in tricuspid valve structure or function. 